\title{
Impactos do climatério em mulheres do sudoeste baiano
}

\author{
Climacteric impact on woman in southwestern of Bahia, Brazil \\ Impactos del climaterio en las mujeres del suroeste de Bahia, Brasil
}

\section{Resumo}

As mulheres ao longo dos anos enfrentam diversas mudanças fisiológicas, relacionadas às distintas fases da sua vida, tais como a menarca, a gestação, ou a última menstruação. O climatério é um processo de transição da fase reprodutiva para não reprodutiva, um acontecimento espontâneo que, segundo a Organização Mundial de Saúde (OMS), ocorre entre os 40 e 65 anos de idade da mulher e envolve diversos aspectos, tais como: endócrinos, físicos, emocionais e socioculturais. $\mathrm{O}$ presente estudo objetiva analisar os principais impactos do climatério na qualidade de vida das mulheres residentes em um município do Sudoeste da Bahia. Trata-se de um estudo de abordagem quantitativa descritiva caracterizada como transversal. A população estudada foi um grupo de 42 mulheres com idade entre 40 e 65 anos. Foi utilizado como instrumento de coleta de dados um questionário semiestruturado dividido em duas partes, a primeira voltada para a identificação do perfil socioeconômico e a segunda composta por questões de abordagem clínica sobre o climatério. Os resultados mostraram a instabilidade do humor e as alterações do sono como os principais fatores que vêm interferindo na qualidade de vida. O perfil das mulheres entrevistadas mostra uma mudança social em relação a estudos anteriores, pois estas possuem independência financeira, cuidam do corpo e tiveram acesso à educação. No entanto, ainda existe uma negligência quanto aos cuidados que devem ser desenvolvidos nesse período.

Palavras-chave: Mulheres; Climatério; Qualidade de vida.

\begin{abstract}
Women over the years face several physiological changes, related to the different phases of their life, such as menarche, pregnancy, or the last menstrual period. Climacteric is a process of transition from the reproductive to the non-reproductive phase, a spontaneous event, which according to the World Health Organization (WHO), occurs between 40 and 65 years of age for women and involves several aspects, such as: endocrine, functional, emotional and socio-cultural. This study aims to analyze the main impacts of climacteric on the quality of life of women living in a southwest city of Bahia. This is a study with a descriptive quantitative approach characterized as cross-sectional. The studied population was a group of 42 women aged between 40 and 65 years. A semi-structured questionnaire divided into two parts was used as a data collection instrument, the first focused on the identification of the socioeconomic profile and the second composed by questions of clinical approach on the climacteric. The results are indispensable to mood instability and as sleep changes as the main factors that interfere in the quality of life. The profile of the women interviewed shows a social change in relation to previous studies, as these are financial independence, take care of the body and had access to education. However, there is still a negligence regarding the care that must be developed during this period.
\end{abstract}

Keywords: Women; Climacteric; Quality of life.

\section{Resumen}

Las mujeres a lo largo de los años se enfrentan a varios cambios fisiológicos, relacionados con las diferentes etapas de su vida, como la menarquia, el embarazo o la última menstruación. El climaterio es un proceso de transición de la fase reproductiva a la no reproductiva, un evento espontáneo, que según la Organización Mundial de la Salud (OMS), ocurre entre los 40 y 65 años de edad para la mujer e involucra varios aspectos, como: endocrino, funcional, 
emocional y sociocultural. Este estudio tiene como objetivo analizar los principales impactos del climaterio en la calidad de vida de las mujeres que viven en un municipio del suroeste de Bahía. Se trata de un estudio de abordaje cuantitativo descriptivo que se caracteriza por ser transversal. La población estudiada fue un grupo de 42 mujeres de entre 40 y 65 años. Se utilizó como instrumento de recolección de datos un cuestionario semiestructurado dividido en dos partes, la primera enfocada a la identificación del perfil socioeconómico y la segunda compuesta por preguntas de abordaje clínico sobre el climaterio. Los resultados son indispensables para la inestabilidad del estado de ánimo y como el sueño cambia como los principales factores que interfieren en la calidad de vida. El perfil de las mujeres entrevistadas muestra un cambio social en relación a estudios previos, ya que estos son independencia económica, cuidado del cuerpo y acceso a la educación. Sin embargo, aún existe una negligencia con respecto a los cuidados que se deben desarrollar durante este período.

Palabras clave: Mujeres; Climatérico; Calidad de vida.

\section{Introdução}

As mulheres ao longo dos anos enfrentam diversas mudanças fisiológicas, relacionadas às distintas fases da sua vida, tais como a menarca, a gestação ou a última menstruação. O aumento da expectativa de vida e o papel social e profissional que conquistaram nos últimos tempos, fizeram com que as mulheres enfrentassem, em plena vida produtiva, um período responsável por grandes transformações metabólicas, hormonais e psicossociais, o chamado climatério (Lomônaco, Tomaz \& Oliveira, 2015). A carência hormonal decorrente da fase climatérica é responsável por grandes alterações no organismo feminino que reduzem, consideravelmente, a qualidade de vida (Assunção, et al., 2017).

O climatério é um processo de transição da fase reprodutiva para não reprodutiva. Esse é um acontecimento espontâneo, que é estabelecido pela Organização Mundial de Saúde (OMS), entre os 40 e 65 anos de idade da mulher e envolve diversos aspectos, como: endócrinos, físicos, emocionais e socioculturais (Diez, et al., 2010). Um marco característico desse período é a menopausa, que se caracteriza pela ocorrência do último ciclo menstrual em consequência da perda da atividade folicular dos ovários e só é reconhecida após 12 meses consecutivos de amenorreia. É relevante para a mulher conhecer e entender sobre todas as mudanças envolvidas nessa passagem, para uma melhor adaptação a essa nova fase da vida (Febrasgo, 2010).

Envelhecer para muitas mulheres é um fator de preocupação e insegurança, seja pelo medo de adoecer ou pela maior consciência do processo de envelhecimento. As mulheres no climatério, especialmente após a menopausa, se sentem menos produtivas e incapazes de manter o ritmo de vida e/ou desempenhar novas atividades, acarretando em interferências nas relações sociais e culturais. Além da interrupção dos ciclos menstruais, é possível que as mulheres apresentem também aumento das taxas de colesterol, doenças cardiovasculares, Diabetes Mellitus, neoplasias benignas e malignas, obesidade, distúrbios urinários, osteoporose e doenças autoimunes (Febrasgo, 2010). Diante de toda sintomatologia e transformações que possam enfrentar neste período, é normal que muitas mulheres apresentem também problemas emocionais (Brasil, 2008).

É importante assegurar que ao longo da vida, a mulher tenha a saúde assistida e acompanhada, pois a informação, a prevenção e o diagnóstico precoce são responsáveis pela diminuição dos agravos (Luiz \& Morais, 2018). Os profissionais de saúde desempenham uma função importante no atendimento dessas mulheres, e conseguem auxiliar na diminuição de complicações durante a fase climatérica através da qualificação da escuta, do acolhimento das queixas e do estímulo ao autocuidado e valorização da vida (Brasil, 2008).

As intervenções mais utilizadas envolvem a adesão a terapias hormonais, não hormonais e não medicamentosas. A reposição hormonal busca recuperar os níveis reduzidos de hormônios por meio de medicamentos alopáticos ou fitoterápicos, visando aliviar os sintomas da menopausa (Silva, et al., 2019). Para algumas mulheres o uso de plantas é uma alternativa segura, principalmente nos casos onde há contraindicação para a terapia hormonal alopática (Rocha, Pereira \& Carneiro, 2018). 
Anteposto, o presente estudo tem como objetivo geral: Analisar os principais impactos do climatério na qualidade de vida das mulheres residentes em um município do Sudoeste da Bahia; e, como objetivos específicos: Analisar a associação entre realizar exames laboratoriais de rotina e os efeitos do climatério; Identificar, por meio de entrevista, as principais alterações físicas e psicológicas provocadas pela fase do climatério em mulheres residentes no sudoeste baiano; Investigar a utilização de fitoterápicos como alternativa terapêutica para os efeitos do climatério.

\section{Metodologia}

Trata-se de um estudo de abordagem quantitativa descritiva caracterizada como transversal (Pereira, et al., 2018). A população estudada foi um grupo de 42 mulheres residentes no sudoeste baiano com idade entre 40 e 65 anos. Visando melhor delineamento da amostra e redução da influência de possíveis fatores externos sobre as variáveis aplicadas, foram considerados como critério de inclusão: ter idade entre 40 e 65 anos, e residir no sudoeste baiano. Para critérios de exclusão foram considerados: estar grávida e relatar uso de medicamento não hormonal para alívio dos sintomas climatéricos.

Para tanto, foi realizada uma busca de dados telefônicos e endereço eletrônico desse público, registrados em um laboratório particular do município Laboratório apoiador, M. L. A. C. LTDA para envio do questionário a ser respondido.

Trata-se de um questionário semiestruturado dividido em duas partes, a primeira voltada para a identificação do perfil socioeconômico da participante, como idade, estado civil, trabalho e renda. A segunda parte do questionário possui questões de abordagem clínica que investigam sobre a ocorrência dos sintomas da menopausa, histórico gestacional, alterações de humor e sono, realização de exames laboratoriais e utilização de medicamentos alopáticos e fitoterápicos. O questionário foi enviado para o e-mail e/ou whatsapp das participantes para aplicação online, através da Plataforma Digital GOOGLE FORMS.

As mulheres que se enquadraram no perfil da população estudada, obedecendo aos critérios de inclusão e exclusão, receberam um link de encaminhamento do GOOGLE FORMS. Nele estavam disponíveis o Termo de Consentimento Livre e Esclarecido (TCLE) e o questionário a ser respondido.

Os dados obtidos por meio das respostas do questionário foram tabulados e codificados em planilha Excel e, em seguida, sendo transcritos para o software SPSS. Com a finalidade de organizar e obter um resumo conciso dos dados, foi utilizada estatística descritiva como análise de frequências absoluta e relativa, cálculos de medidas de tendência central como média, mediana e moda, e de medidas de dispersão como variância e desvio padrão. Os resultados dessa análise foram apresentados em tabelas para melhor visualização dos dados obtidos.

De acordo com a Lei 13.709/2018, referência da regulamentação do tratamento de dados pessoais e pelo cumprimento das Resoluções que norteiam e manipulam as informações médicas, o acesso ao banco de dados ocorreu após a assinatura da Declaração de Autorização para Manipulação de Dados pelo responsável do laboratório fonte e pelos pesquisadores responsáveis pelo estudo. Dessa forma, o desenvolvimento da pesquisa atendeu aos princípios éticos que constam na Resolução No 466/2012 do Conselho Nacional da Saúde (Brasil, 2012) e 510/16 (Brasil, 2016a) do Conselho Nacional de Saúde, sendo realizada após aprovação do Comitê de Ética em Pesquisa da Faculdade Independente do Nordeste (CEP/FAINOR) sob parecer $\mathrm{N}^{\circ}$ 4.613.093.

\section{Resultados e Discussão}

A fase de coleta de dados para esse trabalho ocorreu entre o período de 19 de abril de 2021 a 11 de maio de 2021, com 150 mulheres em idade entre 40 e 65 anos. Dos 150 questionários enviados, 42 foram preenchidos e reenviados por mulheres com idade média de aproximadamente 49 anos e desvio padrão de 6,843. Segundo dados da Pesquisa Nacional por Amostra de Domicílios Contínua (PNAD), realizada em 2019, as faixas etárias com maior estimativa de mulheres no Brasil são de 40 a 44 
anos e 50 a 54 anos (IBGE, 2021). Com base nos dados apresentados, é possível observar que o perfil das mulheres que participaram desse estudo são majoritariamente casadas, com ensino superior completo, vida profissional ativa, não fumantes e praticantes de atividade física regular. Na Tabela 1 está a descrição dos dados socioeconômicos extraídos do Questionário Formulário Socioeconômico:

Tabela 1. Formulário Socioeconômico.

\begin{tabular}{lll}
\hline Variável & Frequência & Percentual \\
\hline Estado Civil & & \\
Solteira & 03 & 7,1 \\
Casada & 28 & 66,7 \\
Divorciada & 04 & 9,5 \\
Outro & 07 & 16,7 \\
Escolaridade & & \\
Ensino Fundamental Incompleto & 05 & 11,9 \\
Ensino Fundamental Completo & 02 & 4,8 \\
Ensino Médio Incompleto & 03 & 7,1 \\
Ensino Médio Completo & 12 & 28,6 \\
Ensino Superior Incompleto & 03 & 7,1 \\
Ensino Superior Completo & 17 & 40,5 \\
Trabalha & & \\
Sim & 31 & 73,8 \\
Não & 11 & 26,2 \\
Renda Familiar & & \\
< 1 salário mínimo & 14 & 33,3 \\
1 a 3 salários mínimos & 13 & 31 \\
> 3 salários mínimos & 15 & 35,7 \\
Fuma & & \\
Sim & 02 & 4,8 \\
Não & 40 & 95,2 \\
Pratica Atividade Física & & \\
1 a 3 vezes por semana & 24 & 42,9 \\
Não pratica & 18 & \\
\hline
\end{tabular}

Fonte: Dados da pesquisa.

Em comparação aos resultados obtidos pelo estudo desenvolvido em 2018 com mulheres quilombolas do nordeste brasileiro, em idade entre 40 e 65 anos, observou-se que o perfil socioeconômico foi de mulheres com até 49 anos, ensino fundamental incompleto, com companheiro estável, situação ocupacional ativa e renda familiar de até 2 salários mínimos (Tavares, et al., 2018).

Epidemiologicamente, as mulheres representam a maioria da população brasileira e formam o principal grupo participativo em ações do Sistema Único de Saúde (SUS) (Brasil, 2008). Em decorrência do crescente envelhecimento populacional, sobretudo em relação às mulheres, estima-se que, de acordo com o censo 2013, haverá um aumento considerável na procura pelos serviços de saúde por mulheres com diferentes queixas relacionadas ao climatério (Assunção, et al, 2017; Moraes \& Schneid, 2015). Apesar de sua etiologia estar atrelada a características genéticas, os efeitos desse período podem ser influenciados por aspectos socioeconômicos, histórico gestacional, tabagismo e alimentação (Febrasgo, 2010).

Desse modo, compete destacar que as alterações físicas e psicológicas provocadas pelo climatério podem influenciar no desempenho e na qualidade de vida desse público potencialmente ativo (Campos, et al., 2013). Além disso, existe a contribuição dos determinates sociais para a saúde dos indivíduos. Dentre estes é possível citar: as condições socioeconômicas e culturais, educação, trabalho, redes sociais, estilo de vida e idade (Assunção, et al., 2017).

Eventos como a menopausa, o natural envelhecimento fisiológico e a decorrente diminuição nas taxas do estrogênio e 
da progesterona, estão associados a diversas repercussões negativas no corpo feminino. Eles estão diretamente associados aos sintomas objetivos do climatério. São eles: as irregularidades menstruais, instabilidade de humor, insônia, osteoporose, doenças cardiovasculares, problemas cognitivos e outros (Filho \& Costa, 2008).

A avaliação clínica no período do climatério é uma estratégia que possibilita identificar precocemante fatores que vêm interferindo na qualidade de vida das mulheres (Figueiredo, et al., 2020). Dentre os achados, a instabilidade do humor representa uma queixa mensal de 42,9\% das entrevistadas, $31 \%$ relata perda de memória e as alterações do sono atinge diarimente 54,8\% desse público. A Tabela 2, demonstrada abaixo, apresenta os dados do questionário, obtidos através da aplicação do formulário clínico:

Tabela 2. Formulário Clínico.

\begin{tabular}{|c|c|c|}
\hline Variável & Frequência & Percentual \\
\hline \multicolumn{3}{|l|}{ Idade da Primeira Menstruação } \\
\hline 8 a 11 anos & 07 & 16,7 \\
\hline 12 a 15 anos & 30 & 71,4 \\
\hline$>15$ anos & 05 & 11,9 \\
\hline \multicolumn{3}{|l|}{ Histórico de Gestações } \\
\hline $\operatorname{Sim}$ & 41 & 97,6 \\
\hline Não & 01 & 2,4 \\
\hline \multicolumn{3}{|l|}{ Histórico de Aborto } \\
\hline Sim & 03 & 7,1 \\
\hline Não & 39 & 92,9 \\
\hline \multicolumn{3}{|l|}{ Histórico de Histerectomia } \\
\hline Sim & 13 & 31 \\
\hline Não & 29 & 69 \\
\hline \multicolumn{3}{|l|}{ Menstrua } \\
\hline Sim & 18 & 42,9 \\
\hline Não & 24 & 57,1 \\
\hline \multicolumn{3}{|l|}{ Tempo sem Menstruar } \\
\hline$<1$ ano & 03 & 12,5 \\
\hline$>1$ ano & 21 & 87,5 \\
\hline \multicolumn{3}{|l|}{ Menopausa } \\
\hline Climatério & 01 & 2,4 \\
\hline Menopausa & 16 & 38,1 \\
\hline Não tem ou não sabe & 25 & 59,5 \\
\hline \multicolumn{3}{|l|}{ Instabilidade de Humor } \\
\hline Diariamente & 11 & 26,2 \\
\hline Mensalmente & 18 & 42,9 \\
\hline Não percebe & 13 & 31 \\
\hline \multicolumn{3}{|l|}{ Alterações no Sono } \\
\hline Diariamente & 23 & 54,8 \\
\hline Mensalmente & 03 & 7,1 \\
\hline Não percebe & 16 & 38,1 \\
\hline \multicolumn{3}{|l|}{ Perda de Memória } \\
\hline Diariamente & 08 & 19 \\
\hline Mensalmente & 05 & 11,9 \\
\hline Não percebe & 29 & 69 \\
\hline \multicolumn{3}{|l|}{ Suores Noturnos } \\
\hline Diariamente & 11 & 26,2 \\
\hline Mensalmente & 05 & 11,9 \\
\hline Não percebe & 26 & 61,9 \\
\hline \multicolumn{3}{|l|}{ Calor Excessivo } \\
\hline Diariamente & 12 & 28,6 \\
\hline Mensalmente & 01 & 2,4 \\
\hline Não percebe & 29 & 69 \\
\hline
\end{tabular}




\begin{tabular}{lll}
\hline Realiza exames com frequência & & \\
Mensalmente & 02 & 4,8 \\
Semestralmente & 13 & 31 \\
Anualmente & 22 & 52,4 \\
Não realiza & 05 & 11,9 \\
Diagnóstico de Doença & & \\
Obesidade & 01 & 2,4 \\
Hipertensão & 12 & 28,6 \\
Outro & 11 & 26,2 \\
Uso de Reposição Hormonal & & \\
Sim & 09 & 21,4 \\
Não & 33 & 78,6 \\
Uso de Fitoterápico & & \\
Sim & 03 & 7,1 \\
Não & 39 & 92,9 \\
\hline
\end{tabular}

Fonte: Dados da pesquisa.

Conforme dados apresentados pelo grupo estudado, 40,5\% relatou estar diagnosticado com menopausa ou em estágio de climatério. Para as mulheres histerectomisadas (31\%), os sinais clínicos do climatério também podem estar presentes, até mesmo pela amenorreia precoce, atrelada à nova condição clínica (Brasil, 2008).

Dentre as 42 entrevistadas, a maioria tem observado instabilidade de humor e alterações no sono (aproximadamente 69\% e 61,9\%, respectivamente), como alguns dos sintomas neuropsíquicos esperados nessa fase da vida reprodutiva (Brasil, 2008). Em estudo realizado em 2006 com mulheres encaminhadas ao Ambulatório de Climatério em Recife, 69,8\% relatou sofrer de distúrbios do sono e 54,3\%, humor reprimido, o que pode interferir diretamente na qualidade das relações e desempenho sociais (Filho \& Costa, 2008).

Esse mesmo estudo observou ainda que mulheres com prática regular de atividade física sofrem menor impacto dos sintomas da menopausa, além de relatarem melhor vigor físico, vitalidade e saúde mental (Filho \& Costa, 2008). Podemos inferir, então, que esse fator pode estar contribuindo para que mais de $60 \%$ das mulheres entrevistadas afirmem não perceber suor noturno e calor excessivo, já que $57,1 \%$ da amostra total relatou praticar atividade física.

Outro dado observado é que, apesar de 59,5\% das entrevistadas relatarem não ter ou não saber informar sobre o diagnóstico de menopausa ou climatério, das 24 mulheres que afirmaram estar com o ciclo menstrual interrompido, 87,5\% diz isso ocorre a mais de 1 ano. Essa lacuna na assistência clínica pode ter refletido no baixo percentual para uso de reposição hormonal (21,4\%) e fitoterápico (7,1\%). Dentre os hormônios citados na pesquisa como em uso, estão: o estradiol, estrogênio, levotiroxina e testosterona, em sua maioria orientados pelo profissional médico. Quanto ao uso de fitoterápicos, o único citado pelas entrevistadas foi a Cápsula de Amora, sob orientação médica ou ato de automedicação.

A terapia de reposição hormonal tem por objetivo compensar a redução em níveis hormonais esperada na fase do climatério, favorecendo para o alívio dos sintomas indesejáveis desse período. Entretanto, sua indicação precisa ser criteriosamente avaliada quanto ao seu risco e benefício. Por isso, a escolha dos fiototerápicos pode ser uma alternativa segura e de eficácia terapêutica comprovada (Petry \& Ramon-Junor, 2012; Brasil, 2016b). Todavia, a sua aceitação popular ainda é influenciada pela crença e tem discreta, porém crescente, aplicação na prática clínica (Rocha, Pereira \& Carneiro, 2018).

Em relação do diagnóstico de doenças, $28,6 \%$ das entrevistadas são hipertensas e 2,4\% tem obesidade. Outros 26,2\% relataram ter diagnóstico de outras doenças como: anemia, hipotireoidismo, trombofilia e Parkinson, por exemplo. Dessa forma, é importante que as mulheres em fase de climatério ou menopausa sejam assistidas clinicamente para que a redução nos níveis de estrogênio esperada nesse período não eleve o risco de surgimento de doenças cardiovasculares e de efeitos negativos sobre o mecanismo de coagulação (Brasil, 2008).

Quando questionadas sobre a realização de exames laboratoriais, 52,4\% afirmou realizá-los anualmente. Das 88,1\% 
que relataram realizar exames laboratoriais com alguma frequência, 45,9\% citou o exame de dosagem do hormônio foliculo estimulante (FSH) e 40,5\% citou, individualmente, os exames de dosagem dos hormônios luteinizante (LH), Estrogênio e Progesterona, considerando que cada participante referiu realizar mais de um desses exames. Esses dados remetem à relevância da assistência clínica para a saúde e qualidade de vida das mulheres durante a fase de climatério, tendo em vista os riscos de adoecimento ocasionados pelas alterações hormonais esperadas nesse período (Dias, et al., 2021; Miranda, Ferreira \& Corrente, 2014).

\section{Conclusão}

O surgimento do climatério é algo que afeta consideravelmente a vida da mulher, pois existem muitas modificações nesse período. Na maioria das vezes, a falta de informação ou de acompanhamento adequado contribui negativamente na qualidade de vida desse grupo. Desse modo, a compreensão global desse período é imprescindível para uma melhor assistência à saúde, pois é necessário não ficar restrito apenas na esfera da sexualidade e da beleza.

$\mathrm{O}$ perfil das mulheres entrevistadas mostra uma mudança social em relação a estudos anteriores, pois são mulheres com independência financeira, que cuidam do corpo e que tiveram acesso à educação. No entanto, ainda existe uma negligência quanto aos cuidados que devem ser desenvolvidos nesse período. Logo, é possível observar que ocorre uma interferência dos sintomas da menopausa na qualidade de vida das entrevistadas.

As alterações do humor e do sono também foram frequentes entre as participantes da pesquisa. De certa forma, isso contribui para a fragilidade das relações sociais construídas, bem como dificulta o início às novas. Dentre os métodos de promoção da qualidade de vida dessas mulheres está a prática de atividade física, citada significativamente pelas entrevistadas e que impacta positivamente nos sinais clássicos do climatério.

O incentivo à educação em saúde é essencial para o bem estar da mulher no período do climatério. Através da informação é possível amenizar as manifestações clínicas e alterações fisiológicas, como as menstruais, emocionais, vasomotoras e corporais. O autocuidado é uma estratégia que deve ser incentivada pelos profissionais de saúde, pois através de medidas terapêuticas, atividade física e cuidados gerais com o corpo é possível manter o bem estar nessa etapa da vida.

Os resultados obtidos nessa pesquisa satisfizeram os objetivos almejados e demonstraram a relevância do tema abordado. Além disso, instigam o interesse sobre trabalhos futuros que abranjam a amostragem em outros serviços de saúde públicos e privados, de modo a possibilitar maior abrangência do público estudado e expandir o potencial de análise sobre esse tema de tamanha relevância para a saúde da mulher.

\section{Referências}

Assunção, D. F. et al. (2017). Qualidade de vida de mulheres climatéricas. Revista da Sociedade Brasileira de Clínica Médica, 15(2), 80-83.

Brasil (2008). Manual de Atenção à Mulher no Climatério/Menopausa.http://bvsms.saude.gov.br/bvs/publicacoes/manual_atencao_mulher_climaterio.pdf.

Brasil (2012). Conselho Nacional de Saúde. Resolução No 466, de 12 de dezembro de 2012. https://conselho.saude.gov.br/resolucoes/2012/Reso466.pdf.

Brasil (2016a). Conselho Nacional de Saúde. Resolução Nº 510, de 07 de abril de 2016. http://conselho.saude.gov.br/resolucoes/2016/Reso510.pdf.

Brasil (2016b). Política $e$ Programa Nacional de Plantas $\quad$ Medicinais $\quad$ Fitoterápicos. http://bvsms.saude.gov.br/bvs/publicacoes/politica_programa_nacional_plantas_medicinais_fitoterapicos.pdf.

Campos, J. C. S., Soares, A. M. B., de Medeiros Cabral, A. L., \& Faustino, C. G. (2013). Uma nova mulher: o climatério e suas alterações fisiopsicoemocionais. In III congresso internacional de envelhecimento humano. Campina grande.

Dias, P. A. R. et al. (2021). Terapia hormonal no climatério como fator de risco para o desenvolvimento de câncer de mama e seus impactos na qualidade de vida. Revista Eletrônica Acervo Saúde, 13(4), e7015-e7015.

Diez, A. C. D. S. B. et al. (2010). Atuação do enfermeiro diante da importância da assistência à saúde da mulher no climatério. Revista mineira de enfermagem, 14(2), 166-174. 
Research, Society and Development, v. 10, n. 7, e22710716563, 2021

(CC BY 4.0) | ISSN 2525-3409 | DOI: http://dx.doi.org/10.33448/rsd-v10i7.16563

Febrasgo (2010). Manual de Orientação Climatério Federação Brasileira das Associações de Ginecologia e Obstetrícia. http://www.itarget.com.br/newclients/sggo.com.br/2008/extra/download/manualCLIMATERIO.

Figueiredo, A. V. et al. (2020). Climatério: perspectivas de mulheres profissionais da saúde do hospital universitário Nova Esperança, João Pessoa-PB. Revista de Ciências da Saúde Nova Esperança, 18(2), 61-72.

Filho, E. A. D., \& Costa, A. M. D. (2008). Avaliação da qualidade de vida de mulheres no climatério atendidas em hospital-escola na cidade do Recife, Brasil. Revista Brasileira de Ginecologia e Obstetrícia, 30(3), 113-120.

Ibge (2021). Instituto Brasileiro de Geografia e Estatística. https://educa.ibge.gov.br/jovens/conheca-o-brasil/populacao/18320-quantidade-de-homens-emulheres.html.

Lomônaco, C., Tomaz, R. A. F., \& de Oliveira Ramos, M. T. (2015). O impacto da menopausa nas relações e nos papéis sociais estabelecidos na família e no trabalho. Reprodução \& Climatério, 30(2), 58-66.

Luiz, L. N. D. S., \& Morais, F. C. D. (2018). Integralidade na assistência à saúde da mulher no climatério.

Miranda, J. S., Ferreira, M. D. L. D. S. M., \& Corrente, J. E. (2014). Qualidade de vida em mulheres no climatério atendidas na Atenção Primária. Revista Brasileira de Enfermagem, 67(5), 803-809.

Moraes, T. O. S., \& Schneid, J. L. (2015). Qualidade de vida no climatério: revisão sistemática da literatura. AMAZÔNIA: SCIENCE \& HEALTH, 3(3), 34-a.

Pereira, A. S. et al. (2018). Metodologia da pesquisa científica.

Petry, K., \& Roman Júnior, W. A. (2012). Viabilidade de implantação de fitoterápicos e plantas medicinais no Sistema Único de Saúde (SUS) do município de Três Passos/RS. Rev Bras Farm, 93(1), 60-7.

Rocha, B. M.A., Pereira, M. D. S. V., \& Carneiro, J. Q. (2018). Terapias complementares: fitoterapia como opção terapêutica no climatério e menopausa. Revista de Ciências da Saúde Nova Esperança, 16(1), 16-25.

Silva, A. S. et al. (2019). O cuidado farmacêutico em mulheres climatéricas e menopáusicas que fazem tratamento farmacológico: uma revisão. VI congresso nacional de envelhecimento humano.

Tavares, A. N. S. et al. (2018). Perfil das mulheres no climatério residentes em uma comunidade quilombola. Revista de Enfermagem UFPE on line, 12(12), 3352-3359. 